Int. J. Dev. Biol. 55: 879-887

doi: $10.1387 / \mathrm{ijdb} .113391 \mathrm{ah}$

\title{
Exosome signaling in mammary gland development and cancer
}

\author{
AN HENDRIX ${ }^{*}, 1$ and ALISTAIR N. HUME ${ }^{2}$ \\ ${ }^{1}$ Laboratory of Experimental Cancer Research, Department of Radiation Oncology and Experimental Cancer \\ Research, Ghent University Hospital, Ghent, Belgium and \\ ${ }^{2}$ School of Biomedical Sciences, Queen's Medical Centre, Nottingham, UK
}

\begin{abstract}
Exosomes are 40-100 $\mathrm{nm}$ intraluminal vesicles that are released by cells upon fusion of multivesicular endosomes (MVEs) with the plasma membrane. The Rab family of small GTPases, including Rab27A and Rab27B, control different steps of exosome release, including transport of MVEs and docking at the plasma membrane. Exosomes are long range message particles that mediate communication between cells in physiological conditions such as mammary gland development and lactation, but also in pathology such as breast cancer. Metastasis is the culmination of cancer progression and involves a complex interaction with the local and distant environment. Exosome messaging contributes to tumor environment interactions such as immune escape, thrombosis and myofibroblast differentiation, thereby modulating metastatic niche preparation.
\end{abstract}

KEY WORDS: exosome, breast cancer, Rab GTPase, exocytosis, metastatic niche

\section{Introduction}

Classically, eukaryotic cells release material into the extracellular environment via the exocytic pathway in which the membranes of Golgi derived vesicles, tubules and granules fuse with the plasma membrane. Cargoes include extracellular matrix (ECM) components, neurotransmitters and peptide growth factors that may be released either constitutively or in response to specific stimuli. Exocytosis is essential for many functions including intercellular signaling and plasma membrane growth. In addition endosomes and lysosomes may undergo processes equivalent to exocytosis and fuse their membranes with the plasma membrane, thereby allowing receptor recycling and plasma membrane repair (Andrews, 2000). While the above pathways allow release of endo-membrane lumen contents and supply components to the plasma membrane, studies of the endocytic trafficking of transferrin receptor in reticulocytes revealed the existence of a distinct exocytic route that releases vesicular carriers of membrane and cytosolic components, known as exosomes, into the extracellular milieu (Pan and Johnstone, 1983). While the role of exosomes in vivo is not well established, they have been the focus of much recent research as in vitro studies suggest that they contribute to physiological and patho-physiological processes including modulation of the immune response, dissemination of viral particles and prions, pathogenesis of neurodegenerative diseases, mediating communication between cancer cells and the environment and transfer of oncogenic signaling receptors to adjacent cells (Simons and Raposo, 2009, Stoorvogel et al., 2002). In this review, we discuss the origin and function of exosomes with particular attention to recent studies of their role in mammary gland development and the pathogenesis of cancer.

\section{Exosomes: definition, origin, composition and destina-} tion

Exosomes are 40-100 $\mathrm{nm}$ diameter extracellular vesicles that have characteristic cup shaped morphology when negatively stained and observed using an electron microscope (Simons and Raposo, 2009, Stoorvogel et al., 2002). They are released by a variety of cells including reticulocytes, cytotoxic T lymphocytes, B lymphocytes, dendritic cells, mast cells, platelets and epithelial cells. Upon release, exosomes circulate in the extracellular space adjacent to the site of discharge where they can be broken down, often within a few minutes. Some of the exosomes, however, can move a distance by diffusion and can appear in biological fluids including blood, urine, amniotic fluid, saliva, lung surfactant, malignant effusions, breast milk and semen. The double-layer mem-

Abbreviations used in this paper: ESCRT, endosomal sorting complex required for transport; ILV, intra-lumenal vesicle; LBPA, lysobisphosphatidic acid; MVE, multivesicular endosome.

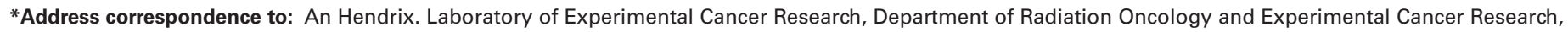
Ghent University Hospital, De Pintelaan 185, 9000 Ghent, Belgium.Tel: +32-9-332-3008. e-mail: an.hendrix@ugent.be
} 
brane, being characterized by a peculiar lipid composition along with protective proteins against complement, makes exosomes more stable than soluble proteins in the extracellular environment (Thery et al., 2002).

Exosomes are thought to originate from the fusion of the membrane of multivesicular endosomes (MVEs, also known as multivesicular bodies (MVBs)) with the plasma membrane. This releases their content of intra-lumenal vesicles (ILVs) into the extracellular space as exosomes (Simons and Raposo, 2009, Stoorvogel et al., 2002). Several pieces of evidence support this idea. Firstly, electron microscopy of B-type Epstein Barr virus infected B-lymphocytes revealed that exosome-like vesicles are released together with endocytosed BSA-gold particles after pulse labeling of MVE (Raposo et al., 1996). Secondly, purified exosomes are enriched in a core set of proteins (e.g. annexins, tetraspanins, flotillins, Rabs, Alix and Tsg101) and lipids (cholesterol, lysobisphosphatidic acid (LBPA) and sphingomyelin) that are abundant in MVEs and are involved in the formation of the ILVs (Simpson et al., 2008, Trajkovic et al., 2008). Interestingly the composition of exosomes appears to differ somewhat depending on their source tissue or cell type. For instance MHC class II is enriched in exosomes from B-lymphocytes, dendritic cells, mast cells, and intestinal epithelial cells while growth factors and their receptors are enriched in exosomes released from cancer cells (Simpson et al., 2008). Thirdly, exosomes contain a variety of highly abundant cytosolic proteins including those involved in structure and motility (tubulin, actin), energy metabolism (e.g. enolase, fatty acid synthase), heat shock response (e.g. hsc70 and hsp90), signal transduction (e.g. 14-3-3 proteins, Ha-Ras) and transcription and protein synthesis (e.g. histones, ribosomal proteins) (Mathivanan et al., 2010, Simpson et al., 2008). In addition to proteins the lumen of exosomes also contain cytosolic RNA. Exosomal messenger (m)RNA and micro (mi)RNA, so called shuttle RNA, can be translated in the exosome's target cell (Valadi et al., 2007). These observations are consistent with the possibility that the process of budding of membrane into the lumen of the endosome, that occurs during the formation of ILVs (see below), results in the capture of abundant cytosolic proteins and RNA within the ILV lumen.

Exosomes have the potential to transfer specific information to both homologous and heterologous target cells. The interaction of exosomes is restricted to defined cells and is determined by exosome composition and the presence of an appropriate ligand on the targeted cell. Several mechanisms of interaction of exosomes with recipient cells have been proposed: cellular binding via receptorligand interaction, attachment or fusion with the target cell membrane, or internalization. These different modes of interaction are not mutually exclusive (Chaput and Thery, 2010, Skog et al., 2008).

\section{Biogenesis of the intra-lumenal vesicles (ILVs) of mul- tivesicular endosomes (MVEs)}

MVEs themselves originate from endosomes that mature and accumulate hundreds of ILVs within their lumen. These vesicles contain endocytosed membrane proteins and lipids e.g. growth factor receptors, and cytosolic proteins destined for degradation following lysosome-MVE fusion (reviewed in Gruenberg and Stenmark, 2004). Indeed most MVEs fuse with lysosome and only a minority are thought to fuse with the plasma membrane to release exosomes. Currently it is unclear what determines which of these fates is met by individual MVEs. In mammalian cells formation of lumenal vesicles occurs via several different pathways meaning that cells may release a range of different exosome types (reviewed in (Hurley et al., 2010) (Fig. 1).

\section{Endosomal sorting complex required for transport (ESCRT)- dependent pathway}

This process is initiated by the presence of membrane tethered ubiquitin, attached to the cytoplasmic tails of endocytosed cell surface receptors destined for degradation, and the lipid phosphatidylinositol 3-phosphate (PI(3)P) on the endosome surface. $\mathrm{PI}(3)$ $P$ is recognized by a FYVE domain containing ESCRT-0 complex sub-unit and recruitment of the other ESCRT-0 sub-units clusters ubiquitinated proteins due to the presence of five ubiquitin binding sites within the complex. Based on the results of in vitro reconstitu-
Fig. 1. Schematic representation of the membrane trafficking pathways that underlie multivesicular endosome formation and exosome release. Plasma membrane components are clustered in budding endocytic vesicles that fuse with early endosomes via a Rab5 dependent mechanism. Early endosome contents are sorted for recycling, degradation and other fates. Cargo destined for degradation is ubiquitinated. Early endosomes mature, accompanied by Rab5 to Rab7 conversion, to multivesicular endosomes (MVE) that contain intralumenal vesicles (ILV). During ILV formation ubiquitinated cargo are clustered into patches in the membrane by the action of ESCRT proteins. ESCRTs promote budding and pinching off of budded membranes resulting in release of vesicles into the endosome lumen. Other ESCRTdependent pathways and ESCRT-independent pathways are discussed in the text. ILV contain membrane proteins, cytosolic proteins and RNA. The majority of MVE then fuse with the lysosomes, resulting in cargo destruction and a subet release their contents to the extracellular space in a Rab27A/Rab27B dependent process.

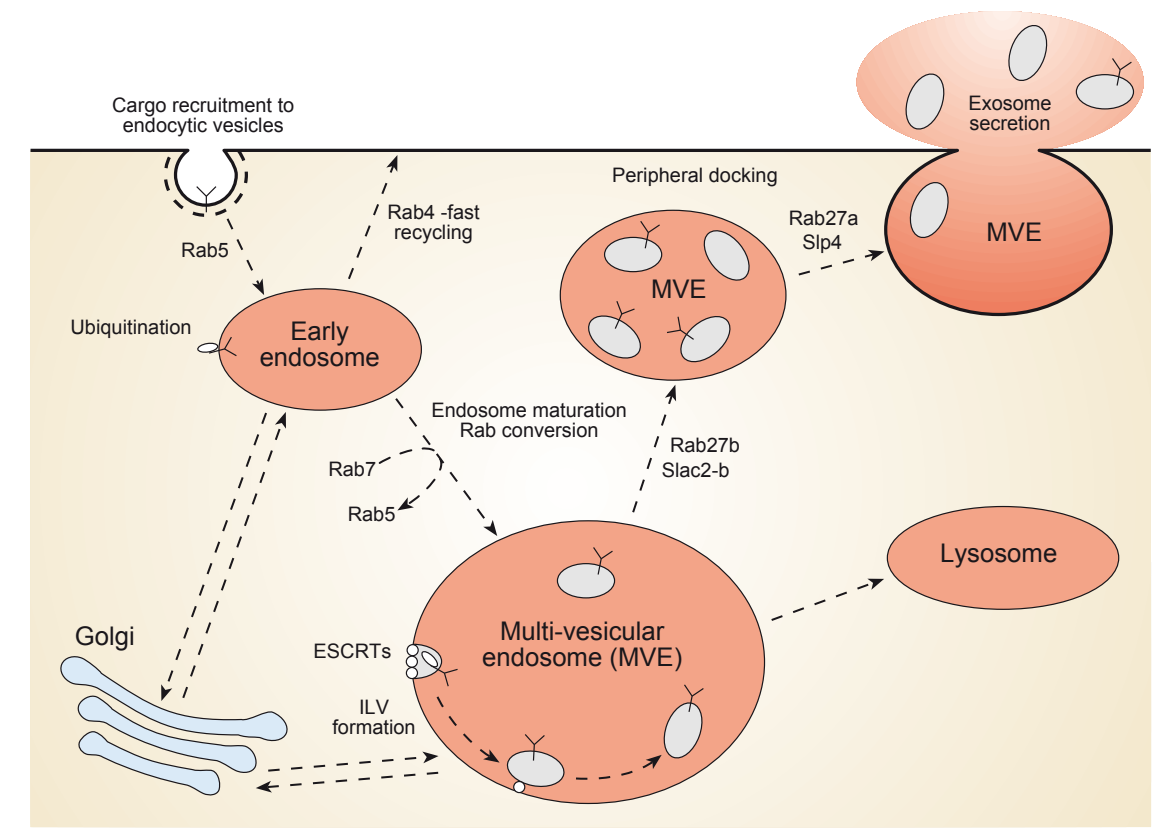


tion experiments, recruitment of ESCRT-0 then initiates recruitment of ESCRT-I and -II complexes, that drives membrane budding, and subsequent ESCRT-III complex recruitment, that cleaves the bud neck to release the vesicle into the lumen (Hurley et al., 2010).

\section{Lysobisphosphatidic acid (LBPA) pathway}

Late endosomes and lysosomes in mammalian cells are enriched $(20 \%)$ in the unusual lipid LBPA that may accumulate and form microdomains on the inner/luminal leaflet of the late endosome membrane due to the acidic luminal $\mathrm{pH}$. This pathway also depends on the activity of the ESCRT proteins (Matsuo et al., 2004).

\section{ESCRT-independent pathways}

Analysis of exosome release by oligodendrocytes in which the ESCRT complex was disabled, by knockdown of its accessory proteins, indicates that proteolipoprotein is sorted into exosomes via a ESCRT-independent pathway. Further analysis of these cells indicated that sphingolipids are enriched in exosomes and that inhibition of sphingomyelinase, that cleaves the phosphodiester bond between ceramide and the sphingomyelin (SM) headgroup, reduces the formation of ILVs in vitro and in cells (Trajkovic et al., 2008). Finally analysis of the melanosomal protein Pmel17 revealed that its trafficking to the ILVs of MVEs was dependent upon the integrity of its lumenal domain and not the ESCRT pathway (Theos et al., 2006).

\section{Rab proteins, and exosome biogenesis and secretion}

Small GTPases of the Rab family regulate cargo and membrane transport through microtubular-and actin-dependent endocytic and secretory pathways (Hutagalung and Novick, 2011, Stenmark, 2009). Specifically Rab5 regulates fusion of endocytic vesicles with early endosomes and homotypic fusion of early endosomes (Spang, 2009). It does this by acting as a compartment specific molecular switch that in the GTP-bound active form recruits cytosolic 'effector' proteins to the early endosome membrane. At the membrane effectors including Vps34-type $\mathrm{PI}(3)$ kinase, early endosome antigen 1 (EEA1) and rabenosyn-5 form a complex with Rab5 together with its GDP/GTP exchange factor Rabex-5. This complex stabilises active Rab5 and promotes further effector recruitment and fusion of endosomes and endocytic vesicles. Briefly, EEA1 dimers are thought to bridge endosomes through $\mathrm{PI}(3)$-P binding FYVE domains located at either end of the molecule and, together with rabenosyn-5 promote homotypic fusion through interaction with SNARE protein syntaxin 6 and SNARE regulator Vps45 (Hutagalung and Novick, 2011, Spang, 2009). The homotypic fusion of early endosomes is interrupted by the recruitment of other Rab proteins involved in the transport of endocytic cargo for recycling (Rab4 and Rab11), degradation (Rab7) or transport to the trans-Golgi network (TGN) (Rab6 and retromer) to other sub-domains of the early endosome network.

Through progressive recycling of endocytosed material to the plasma membrane accompanied by conversion from Rab5- to Rab7-positive status early endosomes mature into late endosomes (Spang, 2009). Indeed sequential recruitment of Rab5 and Rab7 to newly formed endosomes has been observed using live cell imaging (Rink et al., 2005). Recent studies uncovered the ability of active Rab5:GTP to recruit the homotypic fusion and protein sorting (HOPS) complex to early endosomes. HOPS component, Vps39, has Rab7-specific GEF activity and together with other HOPS sub-units stabilise active Rab7:GTP at the membrane resulting in maturation of the compartment to a late endosome (Poteryaev et al., 2010). In spite of this it remains unclearly precisely what factors regulate the timing of effectors recruitment that promote endosome fusion versus those that allow endosome maturation and Rab conversion.

As indicated above little is known about the mechanisms that determine whether MVEs fuse with lysosomes or the plasma membrane. However one recent study identified Rab27 proteins as vital regulators of exosome release (Ostrowski et al., 2010). The Rab27 subfamily comprises two isoforms, Rab27A and Rab27B (71\% amino acid identical) that have been implicated in regulating the exocytosis and transport of a variety of Golgi-derived secretory granules e.g. dense cored secretory granules in PC12 cells, and late endosome/lysosome related secretory granules e.g. melanosomes in melanocytes, lytic granules in T lymphocytes, MIIC in dendritic cells (Izumi, 2007). Using a semi-quantitative FACS-based assay of exosome secretion from HeLa cells stably expressing transactivator CIITA (an activator of the MHC class II family genes, including $H L A-D R$-marker of exosomes) and a secreted form of chicken ovalbumin (marker of the constitutive secretory pathway), they found that RNAi knockdown of several Rabs, including Rab27A and Rab27B, resulted in a specific reduction in exosome secretion. Further analysis showed that knockdown of Rab27B or effector Slac2-b (also known as exophilin 8) resulted in perinuclear clustering of MVEs, marked by ILV associated protein CD63, while knockdown of Rab27A or effector Slp4 (also known as granuphilin) resulted in accumulation of enlarged MVEs. These data suggest that Rab27A and Rab27B play non-redundant roles in the exosomal/MVE secretion pathway via engagement of different effectors. In particular Rab27B and Slac2-b may regulate the transfer of MVEs from microtubule-based transport to actin-based docking at the plasma membrane that is a prelude to exocytosis. Meanwhile Rab27A and SIp4 are suggested to regulate MVE docking and membrane fusion (Ostrowski et al., 2010).

Nevertheless the precise function of these GTPases in MVE transport and exosome secretion together with the mechanism regulating their differential engagement of effectors remains unclear. Moreover, while knockdown of Rab27 proteins (as well as genetic loss of both proteins in mouse) reduced exosome secretion (to $50 \%$ of normal levels) it did not completely block this process, suggesting that other pathways and other Rabs are involved (Ostrowski et al., 2010). Consistent with this Ostrowski and colleagues report that individual knockdown of Rab5A, Rab9A and Rab2 reduced exosome secretion with similar efficiency (Ostrowski et al., 2010). Future studies of these proteins will no doubt provide valuable information on the mechanisms of exosome secretion.

Secretory Rabs not only regulate exocytosis but also exosome release. The role of exosomes in mammary gland development and cancer progression will be discussed in relation to the secretory Rab families Rab3 and Rab27. Our research group recently demonstrated that $\mathrm{Rab} 27 \mathrm{~B}$ regulates invasive growth and metastasis in ER-positive breast cancer lines. Increased expression of Rab27B, but not Rab3D and Rab27A, is associated with poor prognosis in breast tumors (Hendrix et al., 2010).

\section{Exosomes in mammary gland development}

The mammary gland is one of very few organs in which substantial development occurs only after birth. It undergoes cycles 
of growth, differentiation, milk secretion, apoptosis, regression and remodeling during the lifetime of the organism (Bissell et al., 2003). Mammary gland development involves bidirectional interactions between the epithelial population and its surrounding stroma. The stromal environment contributes a depot of adipose tissue, a vasculature that supplies nutrients and endocrine cues, a lymphatic system that not only removes metabolites but also provides an intimate interface with the immune system, and an ECM scaffold, all acting in concert. The epithelium includes a subset of stem cells that closely interact with the environment to alter their fate and the ultimate mammary gland phenotype (Schedin and Hovey, 2010). These key mechanisms involved in normal mammary gland development are hijacked, bypassed or corrupted during the development and progression of cancer.

In the non-pregnant, non-lactating state, the mammary gland consists of networks of epithelial ducts that empty into the main lactiferous ducts. The milk-producing lobular component of the gland is rudimentary, but poised to respond to gestational hormones that elicit local paracrine interactions between the developing epithelial ducts and adjacent stroma. The non-lactating human mammary gland is an actively secreting organ releasing a number of antimicrobial peptides such as $\beta$-defensins, the cathelicidin LL37, lactoferrin and adrenomedullin. These products inhibit bacterial growth in the well-developed duct system. Electron microscopic observations indicate that these peptides are extruded from the glandular cells by secretory granule exocytosis (Welsch etal., 2007). The secretory small GTPase Rab27B, an important regulator of invasive growth in estrogen receptor (ER)-positive breast cancer cells, localizes to the apex of the glandular cells which suggests involvement of this protein in anti-microbial peptide release (Fig. 2) (Hendrix et al., 2010). Furthermore, the non-lactating mammary gland elaborates a prominent glycocalyx at the apical membrane of the glandular epithelial cells. This glycocalyx includes the mucins MUC1 and MUC4, playing an anti-microbial role as well. MUC1 is also released into the lumen and is known to be a constituent of milk in which it protects infants from infections and is highly expressed and aberrantly glycosylated in breast cancer (Patton et al., 1995, Spicer et al., 1995). Mucins can be shed from the plasma membrane by sheddases. An alternative export route using exosome formation and release has been demonstrated for MUC1 in ER-positive MCF-7 breast cancer cells (Staubach et al., 2009). The identification of Rab27B on MVEs and its function in exosome release further suggest a role for the small GTPase in the mammary gland defense mechanism. Lactalbumin and lipid droplets occur also in the non-lactating gland (Welsch et al., 2007).

With pregnancy, and in preparation for lactation, the epithelium undergoes remarkable proliferation and differentiation. The epithelium expands to fill the gland, replacing the fat pad with milkproducing lobuloalveoli. Heregulin beta 1 (HRG), a combinatorial ligand for human epidermal growth factor receptors (HER) 3 and 4 , is expressed in the mammary mesenchyme adjacent to lobuloalveolar structures and is maximally expressed during pregnancy. It plays a role in the morphogenesis and ductal migration of mammary epithelial cells, and promotes the in vitro responsiveness of mammary epithelial cells to lactogenic hormones. HRG stimulation induces the expression of the secretory small GTPase Rab3A, promoting the secretion of cellular proteins from the mammary epithelial cells (Vadlamudi et al., 2000). This nicely illustrates that mammary gland development occurs in an ecosystem where soluble

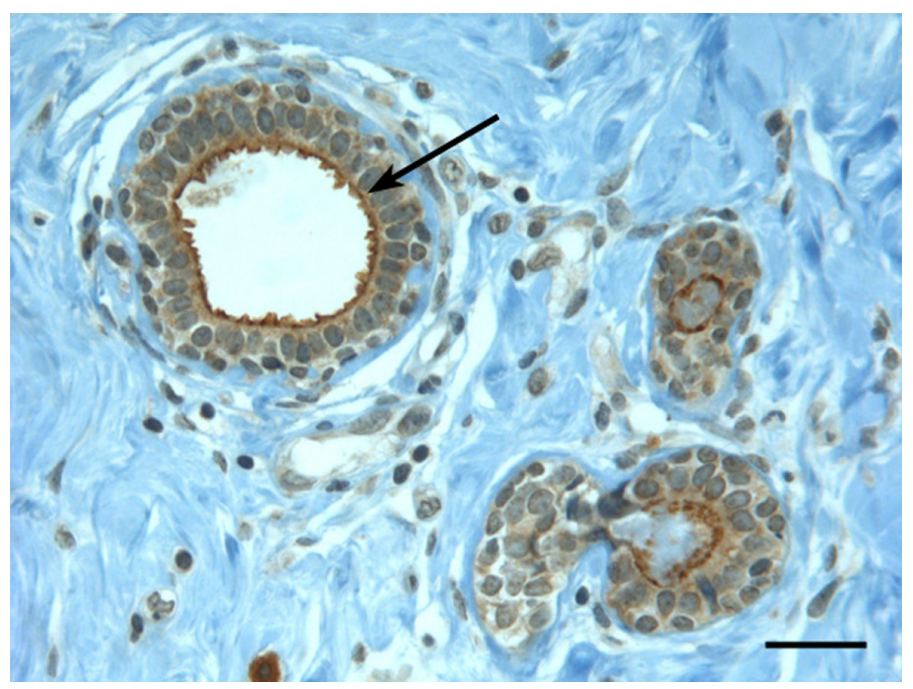

Fig. 2. Rab27B immunostaining in mammary gland. Arrow indicates Rab27B localization to the apex of the glandular cells. Scale bar, $50 \mu \mathrm{m}$.

molecules released by the mammary mesenchyme regulate the secretory tracts in mammary epithelial cells. The effect of HRG on the expression of Rab GTPases involved in exosome release, such as Rab27B, was not investigated.

Breast milk is the optimal nutrition for the newborn infant, containing all the proteins, lipids, carbohydrates, micronutrients and trace elements required for growth, development and immune protection (Jenness, 1979). Furthermore breast milk contains exosomes with the capacity to influence immune responses in the infant. Exosome preparations from colostrums and mature breast milk inhibit CD3-induced interleukin (IL)-2 and interferon (IFN)- $\gamma$, and tumor necrosis factor (TNF)- $\alpha$ production from peripheral blood mononuclear cells (PBMC). An increased number of T-regulatory cells is observed in PBMC incubated with milk vesicle preparations (Admyre et al., 2007).

Lysinuric protein intolerance (LPI) is an autosomal recessive disorder characterized by a marked reduction in the absorption of cationic amino acids leading to low plasma concentrations of lysine, arginine, and ornithine. The impaired transport of cationic amino acids results from mutations in $\mathrm{Y}+\mathrm{L}$ amino acid transporter $1(Y+L A T 1)$ protein found in the basolateral membrane of epithelial cells. Infants with LPI are symptom-free when breastfeed. The induction of other food's leads to a rapid onset of the symptoms associated with the disorder. One possibility is that breast milk offers protection against the primary defect in LPI by the transfer of exosomes containing wild type (maternal) $y+L A T 1$ mRNA to the suckling neonate (Boyd and Shennan, 2010).

With cessation of milk secretion, the mammary gland resorbs the elaborate milk-producing lobuloalveoli generated during pregnancy and returns to its rudimentary, ductal state. As the epithelial cells are lost, the gland is repopulated with adipocytes. Apoptotic mammary epithelial cells must be cleared immediately by phagocytes in involuting mammary glands to prevent inflammation and autoimmune response against intracellular antigens released by dying cells. Apoptotic mammary epithelial cells are phagocytosed by both macrophages and residual living epithelial cells. Exosome-secreted milk fat globule EGF factor 8 (MFG-E8) or lactadherin fulfills a tethering function between apoptotic cells 
and activated macrophages. MFG-E8 is involved in the recognition and clearance of apoptotic mammary epithelial cells during mammary involution (Nakatani et al., 2006). In the mammary glands of MFG-E8 deficient mice impaired involution occurs resulting in periductal mastitis (Hanayama and Nagata, 2005). This severe inflammation probably results from a prolonged involution process in MFG-E8-/- mice with extended activation of inflammatory genes or necrotic cell death. However, mastitis is often caused by bacterial infection and occurs most frequently during early involution. This may be due to a drop in the release of anti-microbial peptidereleasing exosomes that protect the mammary gland from bacterial infiltration. Pregnancy-associated breast cancer, i.e. breast cancer diagnosed within 10 years following parturition, has a higher mortality rate because metastasis is common. A completed pregnancy five-years or less before diagnosis has been demonstrated to be an independent, negative prognostic marker. Since remodeling of the mammary gland to its pre-pregnant state is associated with an influx of immune cells, MMP-dependent ECM reorganization and loss of basement membrane functions with subsequent release of growth factors and bioactive fibronectin fragments, involution is proposed to support metastasis (Schedin, 2006). An important question is whether these local changes form an all-encompassing explanation for an increase in the number of women diagnosed with metastatic disease. Furthermore this hypothesis is built on the assumption of the presence of an invasive microlesion at the time of involution. An alternative hypothesis would be that exosomes released during involution communicate to regional lymph nodes or distant organs, inducing a favorable niche for cancer cells. At the time an occult lesion occurs, transformed epithelial cells home more efficiently and more rapidly to these prepared niches.

\section{Exosomes in cancer}

Cancer is not only a disease of the transformed epithelium but is also influenced and dependent on its stromal environment. Exosomes from both the cancer cells and the stroma determine invasive growth in the primary tumor by local discharge. Some of the exosomes move by diffusion to distant organs to prepare a niche for the settlement and growth of cancer cells.

Cancer cell-derived exosomes modulate invasive growth, adhesion, angiogenesis, immune suppression and chemoresistance

Cancer cells dictate the stromal cell compartment of the primary tumor and mould the ECM to permit invasive growth. Cancer cells deliver transforming growth factor (TGF)- $\beta$ containing exosomes, driving cellular differentiation of fibroblasts, resulting in a myofibroblast phenotype (Webber et al., 2010). Functionally active membrane type (MT)1-matrix metalloprotease (MMP) is secreted in exosomes to degrade type 1 collagen fibers. Invasive breast cancer cells release exosomes containing $\mathrm{HSP} 90 \alpha$, a chaperone required for the activation of extracellular proteases, such as MMP-2 and plasmin (McCready et al., 2010). HER ligands, such as amphiregulin, heparin-binding epidermal growth factor (EGF) and TGF- $\alpha$, are released by cancer exosomes. These ligands are oriented in a signaling-competent manner to engage recipient cell EGF receptor (EGFR) (Higginbotham et al., 2011).

Cancer cells continuously alter their cell surface adhesion molecules allowing to detach from or attach to specific cells, basement laminas and other ECM components, adapting to the dynamic tumor environment encountered during invasion and metastasis. Adhesion molecules are regulated at various levels, including transcription and translation, spatial distribution along the plasma membrane, and endocytosis and degradation. Full-length adhesion molecules are also present in soluble forms outside the cell by exosome secretion and potentially modulate cell adhesion, cell signaling and vesicle-target interaction (van Kilsdonk et al., 2010).

Several studies demonstrated exosome function in angiogenesis, suggesting a role in cancer cell dissemination. Intercellular transfer of a truncated form of EGFR (EGFRvIII) from an EGFRvIIIexpressing cell line to a non-EGFRvlll-expressing cell line regulates VEGF gene expression resulting in angiogenic signaling (Al-Nedawi et al., 2008, Skog et al., 2008). Exosomes dose-dependently stimulate endothelial sprouting and influence endothelial tubule morphology (Hood et al., 2009). Proteomic analysis of secreted exosomes identified potent angiogenic factors such as developmental endothelial locus-1 (DEL-1) and the Notch receptor ligand DII4 (Delta-like 4) (Hegmans et al., 2004, Sheldon et al., 2010). Exosome-induced angiogenesis depends on the tetraspanin D6.1 A (Gesierich et al., 2006).

Exosomes create an immuno-privileged environment within the tumors. Cancer cells produce large amounts of exosomes bearing pro-apoptotic molecules such as Fas ligand and tumor necrosis factor-related apoptosis-inducing (TRAIL) ligand (Huber et al., 2005). These induce apoptosis of activated tumor-specific T cells, impairing the ability of effector lymphocytes to exert their cytolytic activity against tumor targets. Natural killer cells lose their cytolytic potential, through the down-modulation of perforin expression, upon encounter with cancer cell-derived exosomes. Exosomes produced by breast cancer cells block dendritic cell differentiation by increasing IL-6 expression in myeloid precursor cells (Yu et al., 2007). These observations suggest that interfering with exosome release by cancer cells is a novel strategy for recovering multiple immune functions in cancer patients.

Exosomes are important players in chemoresistance. Exosomes released by HER-2 overexpressing breast cancer cell lines SKBR3 and BT474 express activated HER-2. Release of these exosomes is regulated by the growth factors EGF and HRG, two of the known HER-2 receptor cross-activating ligands and naturally present in the surrounding tumor environment. HER-2 positive exosomes act as decoys to inhibit Trastuzumab activity (a humanized monoclonal antibody widely used for the treatment of HER2-overexpressing breastcancer) (Ciravolo etal., 2011). Acquired resistance to cisplatin is associated with abnormalities of protein trafficking and secretion. The lysosomal compartment of human ovarium carcinoma cells selected for stable resistance to cisplatin is markedly reduced in size, and these cells abnormally sort some lysosomal proteins and the putative cisplatin transporters into an exosomal pathway that also exports cisplatin. Enhanced exosomal export was accompanied by higher exosomal levels of the putative cisplatin export transporters MRP2, ATP7A, and ATP7B. This altered distribution of the types of proteins found in exosomes may mark the cisplatin resistant phenotype (Safaei et al., 2005). Because exosomes are found in the systemic circulation, this offers a potential route to early detection of the emergence of chemoresistance during treatment.

Positive feedback mechanisms leading to increased exosome production could be triggered during tumorigenesis. Studies from our group support this idea. The secretory small GTPase Rab27B has 
been described as a key regulator in the transport of multivesicular endosomes and as a consequence exosome release (Ostrowski et al., 2010). Rab27B protein and mRNA are upregulated in poor prognosis ER-positive breast cancer and releases pro-invasive growth regulators in the tumor environment (Hendrix et al., 2010). Wright et al., demonstrated that Rab27B gene expression is estrogen dependent (Wright et al., 2009). Further studies should reveal if Rab27B expression is regulated by additional tumor environment components.

\section{Stromal cell-derived exosomes (in)directly contribute to cancer progression}

Exosomes are not exclusively released by cancer cells. Also the surrounding stromal cells release cell-type specific exosomes contributing to cancer progression.

Pioneering studies by Ratajczak and colleagues demonstrated that exosomes secreted by platelets induce angiogenesis and metastasis in lung and breast cancers (Janowska-Wieczorek et al., 2005). Platelet-derived exosomes increase adhesion of cancer cells to endothelial cells and fibrinogen by transfer to their surface of various platelet-derived integrins such as CD41. Furthermore these exosomes are demonstrated to stimulate the expression of several genes involved in angiogenesis (VEGF, IL-8, HGF) and invasion (MT1-MMP).
Breast cancer cells express antigens confined to the monocyte/ macrophage lineage like CD163, a scavenger receptor. CD163 expression in breast cancer correlates to early distant recurrence. Also, expression of DAP12, a macrophage fusion receptor, in breast cancer is associated with an advanced tumor grade and higher rates of skeletal and liver metastases and a shorter overall disease free survival (Shabo and Svanvik, 2011). These observations suggest a genetic exchange between both cell types by exosome-mediated transfer providing phenotypic characteristics of macrophages such as cell spreading, migration and matrix invasion.

Mast cells are commonly observed in various tumors at the invasive borders, including in breast cancer. Tumor-infiltrating mast cells can directly influence cancer cell proliferation and invasion but also help tumors indirectly by organizing its environment and modulating immune responses to cancer cells (Khazaie et al., 2011). Mast cell-derived exosomes induce plasminogen activator inhibitor type I (PAI-1) expression in endothelial cells (Al-Nedawi et al., 2005). The urokinase-type plasminogen activator (UPA) and the main uPA inhibitor PAI-1 play important roles in cell migration and invasion in both physiological and pathological contexts. Both factors are recommended by the American Society of Clinical Oncology as predictive markers in node-negative breast cancer patients that are used to stratify patients for adjuvant chemotherapy. In addition to their classical functions in plasmin regulation, both factors are key
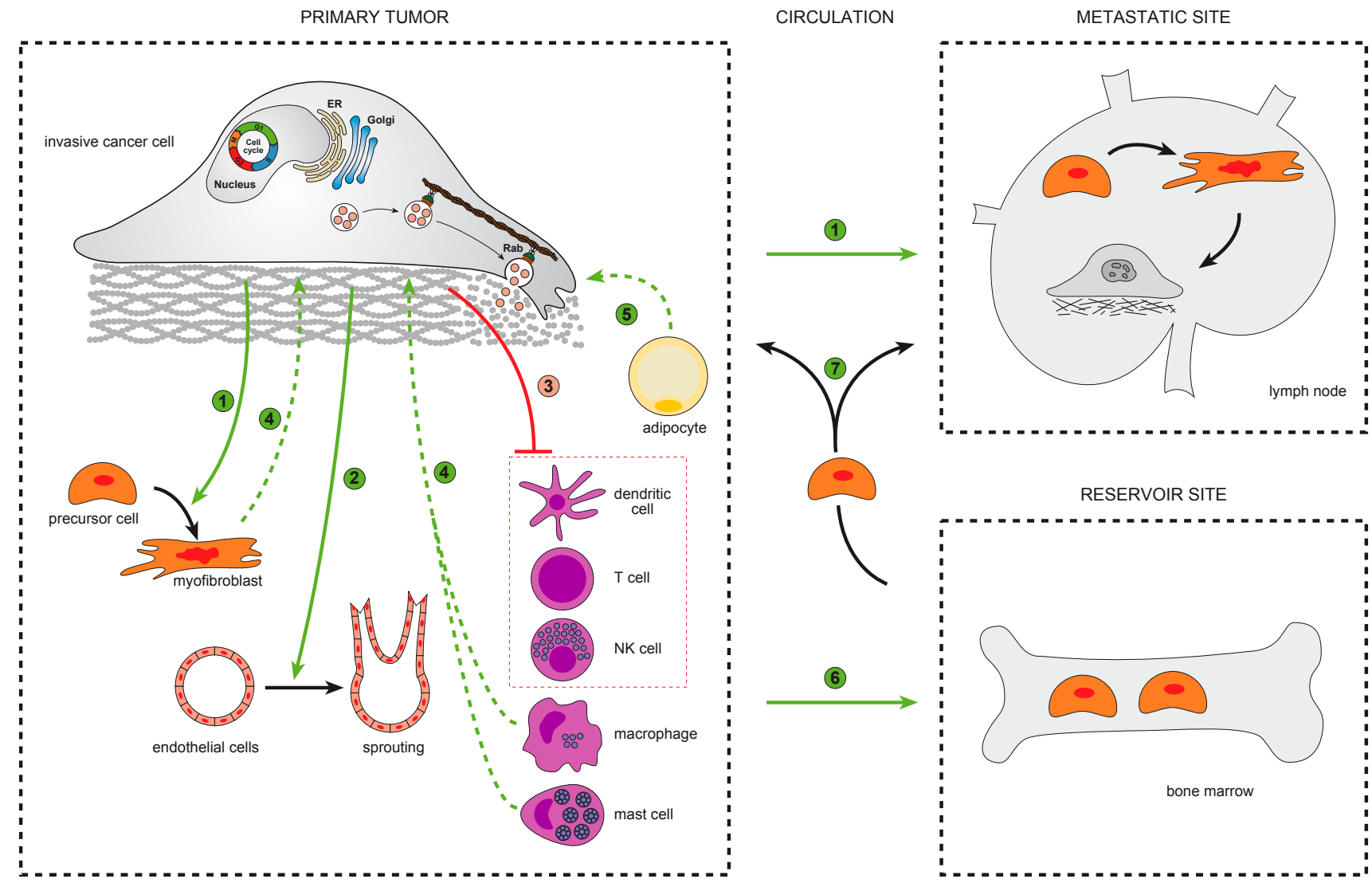

Fig. 3. Effect of cancer cell and stromal cell-derived exosomes on the primary tumor and the distant reservoir and metastatic sites. Cancer-cell derived exosomes trigger myofibroblast differentiation (1), endothelial sprouting (2) and immune suppression (3) in the primary tumor. Cancer cellderived exosomes enter the circulation and home to metastatic sites (e.g. lymph node) to optimize conditions for cancer cell deposition and growth (1). Stromal cell-derived exosomes stimulate invasive growth (4) and cellular metabolism (5). Exosomes secreted by the primary tumor contribute to bone marrow cell education and mobilization (6) and recruit bone marrow progenitor cells to the primary tumor and metastatic sites (7). 
components in cancer-related cell signaling (Harris et al., 2007).

Early local tumor invasion results in immediate proximity of cancer cells to adipocytes. Adipocytes secrete MFG-E8-associated exosomes (Aoki et al., 2007) and adipocyte-derived exosomes have been shown to transfer RNA for lipid synthesis (Muller et al., 2011). Since adipocytes are endocrine cells releasing modulators of multiple metabolic pathways, one hypothesis is that these cells deliver energy to cancer cells for the establishment of invasive growth.

\section{Tumor $\mathrm{pH}$ and hypoxia regulate exosome release}

Some features of the tumor environment may represent key factors in the regulation of exosome traffic within the tumor mass. Tumor acidification, a hallmark of malignancy, is involved in the regulation of some vesicle-mediated malignant cancer cell functions, such as cannibalism and drug resistance (Luciani et al., 2004, Lugini et al., 2006). Acidic $\mathrm{pH}$ results in an increased exosome release and uptake. The increased fusion capacity of exosomes released in an acidic environment is associated to a change in membrane rigidity due to an increased amount of sphingomyelin and ganglioside GM3 lipids. The physiological role of acidity in exosome uptake has been demonstrated for melanoma cells; caveolin-1, a protein involved in melanoma progression, is delivered more efficiently to less aggressive cells in an acidic condition (Parolini et al., 2009). Exosomal shuttle RNA influences the response of recipient cells to an external stress stimulus such as oxidative stress. The mRNA content of exosomes produced under oxidative stress differs extensively from the mRNA in exosomes produced by cells cultured under normal conditions. Exosomes released from mast cells exposed to oxidative stress are shown to have the capacity to communicate a protective signal to recipient cells exposed to oxidative stress, resulting in reduced cell death (Eldh et al., 2010). To sustain growth and survival in their hostile environment, rapidly growing tumors have to overcome hypoxia and a lack of nutrients through either angiogenesis to ensure an adequate supply of oxygen and nutrients or metastasis to a more conductive environment. Under hypoxia, cancer cells secrete exosomes that modulate their local and distant environment to facilitate tumor angiogenesis and metastasis (Park et al., 2010).

\section{Exosomes in the metastatic niche}

Exosomes control metastatic spread whereby routes of communication between the primary tumor and metastatic sites are efficiently maintained. Breast cancer spreads primarily via the lymphatic system. Regional lymph nodes are usually the first metastatic sites to be involved, often followed by distant metastasis to the lungs, liver and bones. Although various prognostic markers are known, regional lymph node status is the single most important prognostic factor in breast cancer. Patients with axillary metastasis at the time of diagnosis have a much worse prognosis. In order to metastasize, cancer cells must manipulate their environment to optimize conditions for deposition and growth both locally and at a distance. Potential sites for remote tumor implantation might thus be prepared well ahead of actual metastasis. Recently an elegant mouse model demonstrated pre-metastatic niche formation in regional lymph nodes through melanoma exosomes. Increased expression of cell recruitment, ECM and endothelial proliferation factors by melanoma exosomes produces a niche within sentinel nodes conductive to melanoma cell recruitment, trapping and growth (Hood et al., 2011).
Bone marrow metastasis of breast cancer can recur even decades after initial diagnosis and treatment, implying the longterm survival of disseminated cancer cells in a dormant state. MDA-MB-231 and T47D breast cancer cells arrest in $\mathrm{G}(0)$ phase of the cell cycle when co-cultured with bone marrow stroma. Breast cancer cell quiescence was induced by stroma-derived exosomes containing miRNAs and specific miRNAs transported from bone marrow stroma to breast cancer cells via gap junctions. This study indicates that exosomes can play a role in the dormancy of bone marrow metastasis (Lim et al., 2011).

\section{Exosomes: targets, biomarkers or therapeutic agents in the clinic}

Tumor exosomes manipulate the metastatic cascade through angiogenesis, stromal remodeling, signal transduction interference by growth factor/receptor transfer, chemoresistance and genetic intercellular exchange. Hence, exosomes offer new opportunities for biomarker analysis and may provide novel specific tools or targets for therapeutic intervention.

Anti-cancer drug resistance results from selective pressure of chemotherapy, together with mutations or epigenetic changes that make cells refractory to treatment. Gene expression associated with vesicle transport correlates with chemosensitivity profiles. Cancer cells implement exosomes as a drug efflux mechanism potentially involved in drug resistance. For example, the cytotoxic drugs doxorubicin and cisplatin are eliminated by exosome-secretion (Shedden et al., 2003). Opposite effects could be expected with drugs interfering with microtubule stability, such as taxanes and vinca alkaloids. In breast cancer cells treated with the microtubule disturbing drug vincistrine, exosome release is reduced (lero et al., 2008). Another promising tool may be represented by drugs interfering with the activity of vacuolar ATPases. Inhibition of this pathway through proton pump inhibitors interferes with the traffic of acidic vesicles, resulting in their sequestration within the cytoplasm, and improving chemosensitivity in cancer cells (Luciani et al., 2004). Alternatively exosome release could be blocked by interfering with the major regulators of multivesicular endosome transport, Rab27A and Rab27B (Ostrowski et al., 2010). Drugs targeting at Rho-associated coiled-coil containing protein kinases (ROCK) affect not only cell morphology, migration and adherence, but also exosome release. Rattan and colleagues showed that inhibition of the Rho/Rock pathway resulted in smaller tumor mass in patients with glioblastoma (Rattan et al., 2006).

Early detection of cancer is vital to improved overall survival rates. As the presence of malignant tumors is clinically determined and/or confirmed upon biopsy procurement - which in itself may have detrimental effects - minimally invasive methods would be highly advantageous to the diagnosis and prognosis of breast cancer and the subsequent tailoring of targeted treatments for individuals. Exosome proteomes of different origins include a common set of membrane and cytosolic proteins, and specific subsets of proteins, likely correlated to cell-type associated functions. This is particularly interesting in relation to their involvement in human diseases. The knowledge of exosome composition can help not only in understanding the biological roles, but also in supplying new biomarkers to be searched for in patients' fluids. In 2005 for the first time, evidence was provided of circulating exosomes in vivo, in blood (Caby et al., 2005). Circulating exosomes have been 
identified as having potential diagnostic relevance in some cancer types including ovarian cancer, glioblastomas and lung cancer (Rabinowits et al., 2009, Skog et al., 2008). The prevalence and clinical relevance of exosomes in sera from breast cancer patients has yet to be elucidated.

Engineered exosomes are emerging as new and novel avenues for cancer vaccine development, via antigen-presenting cell technology, to prime the immune system to recognize and kill cancer cells. An ideal cancer vaccine should be able to prime the immune system to recognize specific tumor antigen and then mount an appropriate immune response toward cancer cells without causing 'by stander effect' damage to neighboring cells. Preclinical studies demonstrated that antigens have higher immunostimulatory capacities when carried by exosomes. To date, three Phase I clinical trials have been conducted, involving the application of exosomes to elicit immune responses against established tumors (Zeelenberg et al., 2008).

\section{Conclusion}

The exosome field needs further exploration in terms of exosome cargo packaging, exosome biogenesis and release mechanisms. The secretory small GTPases, Rab27A and Rab27B, control different steps of exosome release including transport of MVEs and docking at the plasma membrane. Exosome messaging could be involved in long range systemic effects but the specificity of these interactions are poorly understood. Exosomes are implicated in physiology such as mammary gland development and lactation but also in pathology such as breast cancer. Most cancer deaths are due to the development of metastases, hence the most important improvements in morbidity and mortality will result from prevention (or elimination) of such disseminated disease. The potential to specifically inhibit exosome secretion without affecting the regular secretory pathway of soluble proteins paves the way for the manipulation of exosome secretion in vivo, to eventually answer the long-standing question of the involvement of exosomes in metastatic niche preparation and to design therapeutic intervention strategies.

\section{Acknowledgements}

We thank Georges De Bruyne for figure preparations.

\section{References}

ADMYRE, C., JOHANSSON, S.M., QAZI, K.R., FILEN, J.J., LAHESMAA, R., NORMAN, M., NEVE, E.P., SCHEYNIUS, A. and GABRIELSSON, S. (2007). Exosomes with immune modulatory features are present in human breast milk. $J$ Immunol 179: 1969-1978.

AL-NEDAWI, K., MEEHAN, B., MICALLEF, J., LHOTAK, V., MAY, L., GUHA, A. and RAK, J. (2008). Intercellular transfer of the oncogenic receptor EGFRvlll by microvesicles derived from tumour cells. Nat Cell Biol 10: 619-624.

AL-NEDAWI, K., SZEMRAJ, J. and CIERNIEWSKI, C.S. (2005). Mast cell-derived exosomes activate endothelial cells to secrete plasminogen activator inhibitor type 1. Arterioscler Thromb Vasc Biol 25: 1744-1749.

ANDREWS, N.W. (2000). Regulated secretion of conventional lysosomes. Trends Cell Biol 10: 316-321.

AOKI, N., JIN-NO, S., NAKAGAWA, Y., ASAI, N., ARAKAWA, E., TAMURA, N., TAMURA, T. and MATSUDA, T. (2007). Identification and characterization of microvesicles secreted by 3T3-L1 adipocytes: redox- and hormone-dependent induction of milk fat globule-epidermal growth factor 8-associated microvesicles. Endocrinology 148: 3850-3862.

BISSELL, M.J., RIZKI, A. and MIAN, I.S. (2003). Tissue architecture: the ultimate regulator of breast epithelial function. Curr Opin Cell Biol 15: 753-762.
BOYD, C.A. and SHENNAN, D.B. (2010). Breast milk and gene delivery: is lysinuric protein intolerance an exemplar? Mol Genet Metab 101: 296.

CABY, M.P., LANKAR, D., VINCENDEAU-SCHERRER, C., RAPOSO, G. and BONNEROT, C. (2005). Exosomal-like vesicles are present in human blood plasma. Int Immunol 17: 879-887.

CHAPUT, N. and THERY, C. (2011). Exosomes: immune properties and potential clinical implementations. Semin Immunopathol. 33: 419-440.

CIRAVOLO, V., HUBER, V., GHEDINI, G.C., VENTURELLI, E., BIANCHI, F., CAMPIGLIO, M., MORELLI, D., VILLA, A., MINA, P.D., MENARD, S. et al., (2011). Potential role of HER2-overexpressing exosomes in countering Trastuzumab-based therapy. J Cell Physiol. (doi: 10.1002/jcp.22773).

ELDH, M., EKSTROM, K., VALADI, H., SJOSTRAND, M., OLSSON, B., JERNAS, M. and LOTVALL, J. (2010). Exosomes communicate protective messages during oxidative stress; possible role of exosomal shuttle RNA. PLoS One 5: e15353.

GESIERICH, S., BEREZOVSKIY, I., RYSCHICH, E. and ZOLLER, M. (2006). Systemic induction of the angiogenesis switch by the tetraspanin D6.1A/CO-029. Cancer Res 66: 7083-7094.

GRUENBERG, J. and STENMARK, H. (2004). The biogenesis of multivesicular endosomes. Nat Rev Mol Cell Biol 5: 317-323.

HANAYAMA, R. and NAGATA, S. (2005). Impaired involution of mammary glands in the absence of milk fat globule EGF factor 8. Proc Natl Acad Sci USA 102: 16886-16891.

HARRIS, L., FRITSCHE, H., MENNEL, R., NORTON, L., RAVDIN, P., TAUBE, S., SOMERFIELD, M.R., HAYES, D.F. and BAST, R.C., JR. (2007). American Society of Clinical Oncology 2007 update of recommendations for the use of tumor markers in breast cancer. J Clin Oncol 25: 5287-5312.

HEGMANS, J.P., BARD, M.P., HEMMES, A., LUIDER, T.M., KLEIJMEER, M.J., PRINS, J.B., ZITVOGEL, L., BURGERS, S.A., HOOGSTEDEN, H.C. and LAMBRECHT, B.N. (2004). Proteomic analysis of exosomes secreted by human mesothelioma cells. Am J Pathol 164: 1807-1815.

HENDRIX, A., MAYNARD, D., PAUWELS, P., BRAEMS, G., DENYS, H., VAN DEN BROECKE, R., LAMBERT, J., VAN BELLE, S., COCQUYT, V., GESPACH, C. et al., (2010). Effect of the secretory small GTPase Rab27B on breast cancer growth, invasion, and metastasis. J Natl Cancer Inst 102: 866-880.

HIGGINBOTHAM, J.N., DEMORY BECKLER, M., GEPHART, J.D., FRANKLIN, J.L., BOGATCHEVA, G., KREMERS, G.J., PISTON, D.W., AYERS, G.D., MCCONNELL, R.E., TYSKA, M.J. et al., (2011). Amphiregulin exosomes increase cancer cell invasion. Curr Biol 21: 779-786.

HOOD, J.L., PAN, H., LANZA, G.M. and WICKLINE, S.A. (2009). Paracrine induction of endothelium by tumor exosomes. Lab Invest 89: 1317-1328.

HOOD, J.L., ROMAN, S.S. and WICKLINE, S.A. (2011). Exosomes Released by Melanoma Cells Prepare Sentinel Lymph Nodes for Tumor Metastasis. Cancer Res. 71: 3792-3801.

HUBER, V., FAIS, S., IERO, M., LUGINI, L., CANESE, P., SQUARCINA, P., ZACCHEDDU, A., COLONE, M., ARANCIA, G., GENTILE, M. et al., (2005). Human colorectal cancer cells induce T-cell death through release of proapoptotic microvesicles: role in immune escape. Gastroenterology 128: 1796-1804.

HURLEY, J.H., BOURA, E., CARLSON, L.A. and ROZYCKI, B. (2010). Membrane budding. Cell 143: 875-887.

HUTAGALUNG, A.H. and NOVICK, P.J. (2011). Role of Rab GTPases in membrane traffic and cell physiology. Physiol Rev 91: 119-149.

IERO, M., VALENTI, R., HUBER, V., FILIPAZZI, P., PARMIANI, G., FAIS, S. and RIVOLTINI, L. (2008). Tumour-released exosomes and their implications in cancer immunity. Cell Death Differ 15: 80-88.

IZUMI, T. (2007). Physiological roles of Rab27 effectors in regulated exocytosis. Endocr J 54: 649-657.

JANOWSKA-WIECZOREK, A., WYSOCZYNSKI, M., KIJOWSKI, J., MARQUEZCURTIS, L., MACHALINSKI, B., RATAJCZAK, J. and RATAJCZAK, M.Z. (2005). Microvesicles derived from activated platelets induce metastasis and angiogenesis in lung cancer. Int J Cancer 113: 752-760.

JENNESS, R. (1979). The composition of human milk. Semin Perinatol 3: 225-239 KHAZAIE, K., BLATNER, N.R., KHAN, M.W., GOUNARI, F., GOUNARIS, E., DENNIS, K., BONERTZ, A., TSAI, F.N., STROUCH, M.J., CHEON, E. et al., (2011). The significant role of mast cells in cancer. Cancer Metastasis Rev 30: 45-60.

LIM, P.K., BLISS, S.A., PATEL, S.A., TABORGA, M., DAVE, M.A., GREGORY, L.A., 
GRECO, S.J., BRYAN, M., PATEL, P.S. and RAMESHWAR, P. (2011). Gap junction-mediated import of microRNA from bone marrow stromal cells can elicit cell cycle quiescence in breast cancer cells. Cancer Res 71: 1550-1560.

LUCIANI, F., SPADA, M., DE MILITO, A., MOLINARI, A., RIVOLTINI, L., MONTINARO, A., MARRA, M., LUGINI, L., LOGOZZI, M., LOZUPONE, F. et al., (2004). Effect of proton pump inhibitor pretreatment on resistance of solid tumors to cytotoxic drugs. J Natl Cancer Inst 96: 1702-1713.

LUGINI, L., MATARRESE, P., TINARI, A., LOZUPONE, F., FEDERICI, C., IESSI, E., GENTILE, M., LUCIANI, F., PARMIANI, G., RIVOLTINI, L. et al., (2006). Cannibalism of live lymphocytes by human metastatic but not primary melanoma cells. Cancer Res 66: 3629-3638.

MATHIVANAN, S., JI, H. and SIMPSON, R.J. (2010). Exosomes: extracellular organelles important in intercellular communication. J Proteomics 73: 1907-1920.

MATSUO, H., CHEVALLIER, J., MAYRAN, N., LE BLANC, I., FERGUSON, C., FAURE, J., BLANC, N.S., MATILE, S., DUBOCHET, J., SADOUL, R. et al., (2004). Role of LBPA and Alix in multivesicular liposome formation and endosome organization. Science 303: 531-534.

MCCREADY, J., SIMS, J.D., CHAN, D. and JAY, D.G. (2010). Secretion of extracellular hsp90alpha via exosomes increases cancer cell motility: a role for plasminogen activation. BMC Cancer 10: 294.

MULLER, G., SCHNEIDER, M., BIEMER-DAUB, G. and WIED, S. (2011). Microvesicles released from rat adipocytes and harboring glycosylphosphatidylinositol-anchored proteins transfer RNA stimulating lipid synthesis. Cell Signal 23: 1207-1223.

NAKATANI, H., AOKI, N., NAKAGAWA, Y., JIN-NO, S., AOYAMA, K., OSHIMA, K., OHIRA, S., SATO, C., NADANO, D. and MATSUDA, T. (2006). Weaning-induced expression of a milk-fat globule protein, MFG-E8, in mouse mammary glands, as demonstrated by the analyses of its mRNA, protein and phosphatidylserinebinding activity. Biochem J 395: 21-30.

OSTROWSKI, M., CARMO, N.B., KRUMEICH, S., FANGET, I., RAPOSO, G., SAVINA, A., MOITA, C.F., SCHAUER, K., HUME, A.N., FREITAS, R.P. et al., (2010). Rab27a and Rab27b control different steps of the exosome secretion pathway. Nat Cell Biol 12: 19-30.

PAN, B.T. and JOHNSTONE, R.M. (1983). Fate of the transferrin receptor during maturation of sheep reticulocytes in vitro: selective externalization of the receptor. Cell 33: 967-978.

PARK, J.E., TAN, H.S., DATTA, A., LAI, R.C., ZHANG, H., MENG, W., LIM, S.K. and SZE, S.K. (2010). Hypoxic tumor cell modulates its microenvironment to enhance angiogenic and metastatic potential by secretion of proteins and exosomes. Mol Cell Proteomics 9: 1085-1099.

PAROLINI, I., FEDERICI, C., RAGGI, C., LUGINI, L., PALLESCHI, S., DE MILITO, A., COSCIA, C., IESSI, E., LOGOZZI, M., MOLINARI, A. et al., (2009). Microenvironmental $\mathrm{pH}$ is a key factor for exosome traffic in tumor cells. $\mathrm{J} \mathrm{Biol} \mathrm{Chem}$ 284: 34211-34222.

PATTON, S., GENDLER, S.J. and SPICER, A.P. (1995). The epithelial mucin, MUC1, of milk, mammary gland and other tissues. Biochim Biophys Acta 1241: 407-423.

POTERYAEV, D., DATTA, S., ACKEMA, K., ZERIAL, M. and SPANG, A. (2010). Identification of the switch in early-to-late endosome transition. Cell 141:497-508.

RABINOWITS, G., GERCEL-TAYLOR, C., DAY, J.M., TAYLOR, D.D. and KLOECKER, G.H. (2009). Exosomal microRNA: a diagnostic marker for lung cancer. Clin Lung Cancer 10: $42-46$

RAPOSO, G., NIJMAN, H.W., STOORVOGEL, W., LIEJENDEKKER, R., HARDING, C.V., MELIEF, C.J. and GEUZE, H.J. (1996). B lymphocytes secrete antigenpresenting vesicles. J Exp Med 183: 1161-1172.

RATTAN, R., GIRI, S., SINGH, A.K. and SINGH, I. (2006). Rho/ROCK pathway as a target of tumor therapy. $J$ Neurosci Res 83: 243-255.

RINK, J., GHIGO, E., KALAIDZIDIS, Y. and ZERIAL, M. (2005). Rab conversion as a mechanism of progression from early to late endosomes. Cell 122: 735-749.

SAFAEI, R., LARSON, B.J., CHENG, T.C., GIBSON, M.A., OTANI, S., NAERDEMANN, W. and HOWELL, S.B. (2005). Abnormal lysosomal trafficking and enhanced exosomal export of cisplatin in drug-resistant human ovarian carcinoma cells. Mol Cancer Ther 4: 1595-1604.

SCHEDIN, P. (2006). Pregnancy-associated breast cancer and metastasis. Nat Rev Cancer 6: 281-291.

SCHEDIN, P. and HOVEY, R.C. (2010). Editorial: The mammary stroma in normal development and function. J Mammary Gland Biol Neoplasia 15: 275-277.
SHABO, I. and SVANVIK, J. (2011). Expression of macrophage antigens by tumor cells. Adv Exp Med Biol 714: 141-150.

SHEDDEN, K., XIE, X.T., CHANDAROY, P., CHANG, Y.T. and ROSANIA, G.R. (2003). Expulsion of small molecules in vesicles shed by cancer cells: association with gene expression and chemosensitivity profiles. Cancer Res 63: 4331-4337.

SHELDON, H., HEIKAMP, E., TURLEY, H., DRAGOVIC, R., THOMAS, P., OON, C.E., LEEK, R., EDELMANN, M., KESSLER, B., SAINSON, R.C. et al., (2010). New mechanism for Notch signaling to endothelium at a distance by Delta-like 4 incorporation into exosomes. Blood 116: 2385-2394.

SIMONS, M. and RAPOSO, G. (2009). Exosomes--vesicular carriers for intercellular communication. Curr Opin Cell Biol 21: 575-581.

SIMPSON, R.J., JENSEN, S.S. and LIM, J.W. (2008). Proteomic profiling of exosomes: current perspectives. Proteomics 8: 4083-4099.

SKOG, J., WURDINGER, T., VAN RIJN, S., MEIJER, D.H., GAINCHE, L., SENAESTEVES, M., CURRY, W.T., JR., CARTER, B.S., KRICHEVSKY, A.M. and BREAKEFIELD, X.O. (2008). Glioblastoma microvesicles transport RNA and proteins that promote tumour growth and provide diagnostic biomarkers. Nat Cell Biol 10: 1470-1476.

SPANG, A. (2009). On the fate of early endosomes. Biol Chem 390: 753-759.

SPICER, A.P., ROWSE, G.J., LIDNER, T.K. and GENDLER, S.J. (1995). Delayed mammary tumor progression in Muc-1 null mice. J Biol Chem 270: 30093-30101.

STAUBACH, S., RAZAWI, H. and HANISCH, F.G. (2009). Proteomics of MUC1containing lipid rafts from plasma membranes and exosomes of human breast carcinoma cells MCF-7. Proteomics 9: 2820-2835.

STENMARK, H. (2009). Rab GTPases as coordinators of vesicle traffic. Nat Rev Mol Cell Biol 10: 513-525.

STOORVOGEL, W., KLEIJMEER, M.J., GEUZE, H.J. and RAPOSO, G. (2002). The biogenesis and functions of exosomes. Traffic 3: 321-330.

THEOS, A.C., TRUSCHEL, S.T., TENZA, D., HURBAIN, I., HARPER, D.C., BERSON, J.F., THOMAS, P.C., RAPOSO, G. and MARKS, M.S. (2006). A lumenal domain-dependent pathway for sorting to intralumenal vesicles of multivesicular endosomes involved in organelle morphogenesis. Dev Cell 10: 343-354.

THERY, C., ZITVOGEL, L. and AMIGORENA, S. (2002). Exosomes: composition, biogenesis and function. Nat Rev Immunol 2: 569-579.

TRAJKOVIC, K., HSU, C., CHIANTIA, S., RAJENDRAN, L., WENZEL, D., WIELAND, F., SCHWILLE, P., BRUGGER, B. and SIMONS, M. (2008). Ceramide triggers budding of exosome vesicles into multivesicular endosomes. Science 319: 1244-1247.

VADLAMUDI, R.K., WANG, R.A., TALUKDER, A.H., ADAM, L., JOHNSON, R. and KUMAR, R. (2000). Evidence of Rab3A expression, regulation of vesicle trafficking, and cellular secretion in response to heregulin in mammary epithelial cells. Mol Cell Biol 20: 9092-9101.

VALADI, H., EKSTROM, K., BOSSIOS, A., SJOSTRAND, M., LEE, J.J. and LOTVALL, J.O. (2007). Exosome-mediated transfer of mRNAs and microRNAs is a novel mechanism of genetic exchange between cells. Nat Cell Biol 9: 654-659.

VAN KILSDONK, J.W., VAN KEMPEN, L.C., VAN MUIJEN, G.N., RUITER, D.J. and SWART, G.W. (2010). Soluble adhesion molecules in human cancers: sources and fates. Eur J Cell Biol 89: 415-427.

WEBBER, J., STEADMAN, R., MASON, M.D., TABI, Z. and CLAYTON, A. (2010). Cancer exosomes trigger fibroblast to myofibroblast differentiation. Cancer Res 70: 9621-9630.

WELSCH, U., OPPERMANN, T., MORTEZZA, M., HOFTER, E. and UNTERBERGER, P. (2007). Secretory phenomena in the non-lactating human mammary gland. Ann Anat 189: 131-141.

WRIGHT, P.K., MAY, F.E., DARBY, S., SAIF, R., LENNARD, T.W. and WESTLEY, B.R. (2009). Estrogen regulates vesicle trafficking gene expression in EFF-3, EFM-19 and MCF-7 breast cancer cells. Int. J. Clin. Exp. Pathol. 2: 463-475.

YU, S., LIU, C., SU, K., WANG, J., LIU, Y., ZHANG, L., LI, C., CONG, Y., KIMBERLY, R., GRIZZLE, W.E. et al., (2007). Tumor exosomes inhibit differentiation of bone marrow dendritic cells. J Immunol 178: 6867-6875.

ZEELENBERG, I.S., OSTROWSKI, M., KRUMEICH, S., BOBRIE, A., JANCIC, C., BOISSONNAS, A., DELCAYRE, A., LE PECQ, J.B., COMBADIERE, B., AMIGORENA, S. et al., (2008). Targeting tumor antigens to secreted membrane vesicles in vivo induces efficient antitumor immune responses. Cancer Res 68: 1228-1235. 


\section{Further Related Reading, published previously in the Int. J. Dev. Biol.}

Rab11 is required for cell adhesion, maintenance of cell shape and actin-cytoskeleton organization during Drosophila wing development

Tanmay Bhuin and Jagat K. Roy

Int. J. Dev. Biol. (2011) 55: 269-279

Modeling of angioadaptation: insights for vascular development

Axel R. Pries, Bettina Reglin and Timothy W. Secomb

Int. J. Dev. Biol. (2011) 55: 399-405

Trisomy 21- affected placentas highlight prerequisite factors for human trophoblast fusion and differentiation André Malassiné, Jean-Louis Frendo and Danièle Evain-Brion

Int. J. Dev. Biol. (2010) 54: 475-482

Casein kinase I epsilon somatic mutations found in breast cancer cause overgrowth in Drosophila

Tomas Dolezal, Katerina Kucerova, Jana Neuhold and Peter J. Bryant

Int. J. Dev. Biol. (2010) 54: 1419-1424

Bmp4-directed nuclear cyan fluorescent protein provides a tool for live imaging and reveals cellular resolution of Bmp4 expression patterns during embryogenesis

Chuan-Wei Jang, Liang Gao, Mary E. Dickinson and Richard R. Behringer

Int. J. Dev. Biol. (2010) 54: 931-938

Generation of a reporter-null allele of Ppap2b/Lpp3and its expression during embryogenesis Diana Escalante-Alcalde, Sara L. Morales and Colin L. Stewart

Int. J. Dev. Biol. (2009) 53: 139-147

Epithelial-Mesenchymal Transitions in development and disease: old views and new perspectives

M. Angela Nieto

Int. J. Dev. Biol. (2009) 53: 1541-1547

Genetic control of morphogenesis - Hox induced organogenesis of the posterior spiracles James Castelli-Gair Hombría, María Luisa Rivas and Sol Sotillos

Int. J. Dev. Biol. (2009) 53: 1349-1358

Oyster sperm bindin is a combinatorial fucose lectin with remarkable intra-species diversity Stevan A. Springer, Gary W. Moy, Daniel S. Friend, Willie J. Swanson and Victor D. Vacquier Int. J. Dev. Biol. (2008) 52: 759-768

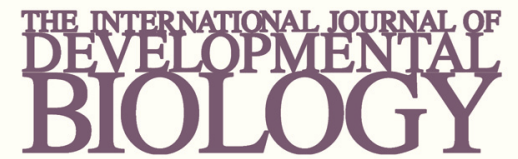

Volume 54 Nos. 6/7
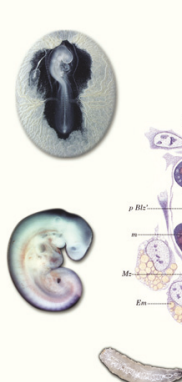

Developmental Hematopoiesis
Special Issue
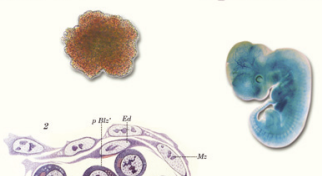

(2)
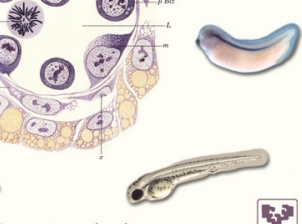

虚
5 yr ISI Impact Factor $(2010)=2.961$
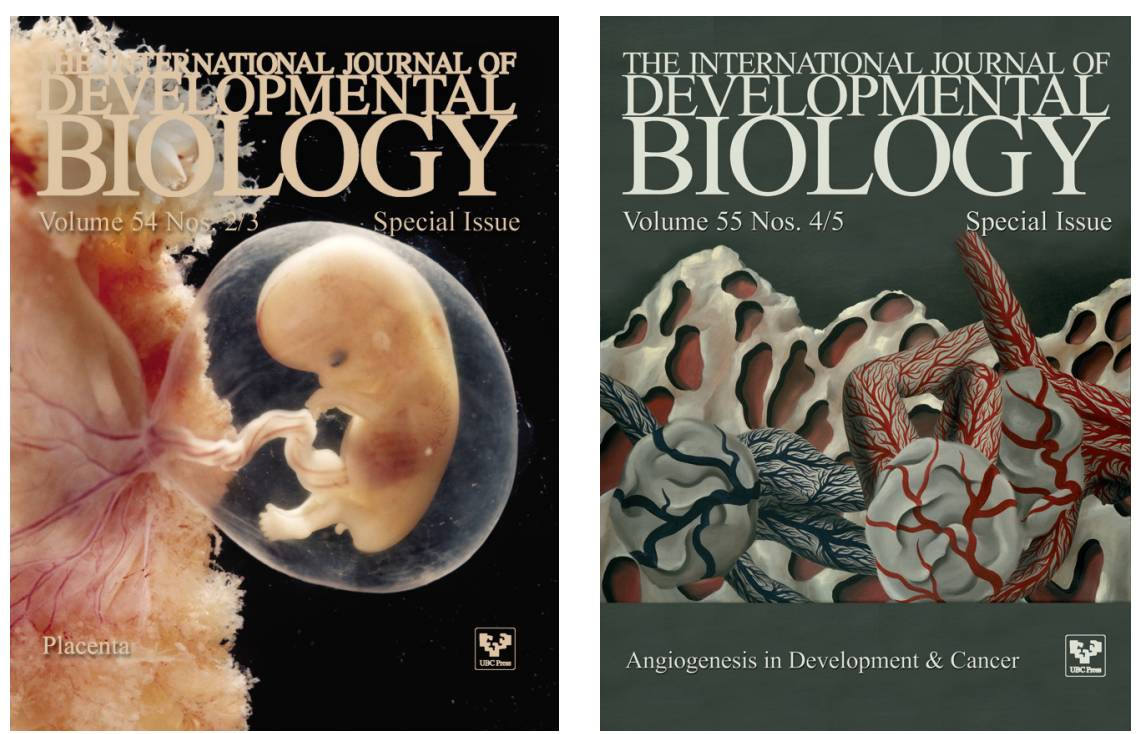